\title{
A Microarray Analysis of Parkinson's Disease: New Clues and Evaluation
}

\author{
Lirong Huo ${ }^{1,2}$, Xibin Liang3, Yi He2, Xiaomin Wang2* \\ ${ }^{1}$ Department of Neurology, Fu Xing Hospital, Capital Medical University, Beijing, China \\ ${ }^{2}$ Department of Physiology, Key Laboratory for Neurodegenerative Disorders of the Ministry of Education, \\ Captal Medical University, Beijing, China \\ ${ }^{3}$ Department of Neurology, Stanford University, Stanford, USA \\ Email: huolr002@163.com, x.xwwang@ccmu.edu.cn
}

Received 31 July 2015; accepted 10 September 2015; published 17 September 2015

\begin{abstract}
Parkinson's disease (PD) is complex and most likely results from an unknown combination of genetic and environmental factors. Here, we defined discrete genes (DGs) in a microarray analysis and found that the percentage of DGs versus all analyzable genes correlated with PD progression. Furthermore, this new parameter was also easily used to evaluate the therapeutic effect of highfrequency electro-acupuncture (EA), thus improving symptoms of PD model rats.
\end{abstract}

\section{Keywords}

Parkinson's Disease, 6-0HDA, Microarray, Discrete Genes, Cortex, Striatum

\section{Introduction}

In the past two decades, rapid advances in gene expression profiling using microarray technology have not only brightened the prospect of deciphering the complexity of the disease genesis and progression at the genomic level but have also revolutionized the diagnostic, therapeutic, and prognostic approaches [1]. Therefore, to improve our understanding of biological regulation, we need to approach the inherent biological complexity of gene expression with a network-based approach to systems biology [2]. Even if the whole population of cells has been thought to be identical, each one has a different expression profile [3]. Faced with thousands of differential genes, we are often unable to quickly assess the severity of some complex disorders without a precise parameter based on array analysis. Parkinson's disease (PD) is characterized by the progressive degeneration of central neural pathways from the dorsal motor nuclei, to the hallmark DAergic neurons of the substantia nigra (SN), and finally, to additional regions such as the neocortex [4]. The cause of PD is unknown, but a complex mixture of genetic and environmental risk factors has been thought to be the cause in many cases (Heidi Ledford, Nature news, 2010). Analysis of microarray data is becoming much easier due to the availability free online databases, such as David 6.7 [5] [6]. The dysregulation of numerous genes in the SN from both PD animal models and human patients has been identified using microarray assays [7]. However, the precise roles of the altered

${ }^{*}$ Corresponding author. 
gene expressions remain unclear, and the distinct relationships between genes must be identified.

\section{Methods}

With this objective, we used microarrays to explore the gene expression profiles in the 6-OHDA-unilateral lesion rat model of PD between two phases (28ds and 35ds). The experimental procedures were approved by the Committee on Animal Care and Usage, Capital Medical University, and all efforts were made to minimize animal suffering. An injection of 6-OHDA into the medial forebrain bundle (MFB lesion model) causes effects similar to end-stage PD [8]. The number of rotations observed after amphetamine administration has been shown to distinguish between partial and near complete $(>90 \%)$ denervation in the SN. Four weeks 6-OHDA treatment, a nearly complete denervation in the rat SN was observed in our experiments (data not shown). First, microarray analysis was carried out to investigate gene expression patterns in the cortex and striatum (STR). 6-OHDA-targeted transcripts in the Cortex and STR were analyzed with Whole Rat Genome $4 \times 44 \mathrm{~K}$ microarrays (Gene expression hybridization kit, 5188 - 5242, Agilent). Three biological replicates from each group were analyzed using an Agilent cDNA microarray. Overall, many differentially expressed genes were found in the model cortex and STR up to 4 weeks and 5 weeks after 6-OHDA treatment when compared to the control. Then, when mutual genes were deleted from the two compared groups, we had also found many differentially expressed genes in the cortex or STR of the model 4-/5-week group compared to the model 5-/4-week group. Subsequently, we performed high throughput microarray analyses to evaluate the effect of EA and integrative data mining to uncover the progression of gene profile changes in the cortex and STR. Also, three biological replicates from each group were analyzed (See Figure 1). The Model group was analyzed 5 weeks after 6-OHDA injections into the unilateral MFB. The EA group was treated using 100-Hz EA for 4 weeks.

\section{Results and Discussion}

\subsection{Differentially Expressed Transcripts and Discrete Genes (DG) Levels in the Cortex and STR Targeted by 6-0HDA}

Including the unknown genes, the cortex chips showed 1289 differential transcripts (690 up-regulated and 599 down-regulated, $\mathrm{p}<0.05)$ after 4 weeks of 6-OHDA treatment in the model group and 912 differential transcripts (507 up-regulated and 405 down-regulated, $\mathrm{p}<0.05$ ) after 5 weeks $(5 \mathrm{~W})$ compared to the control. To

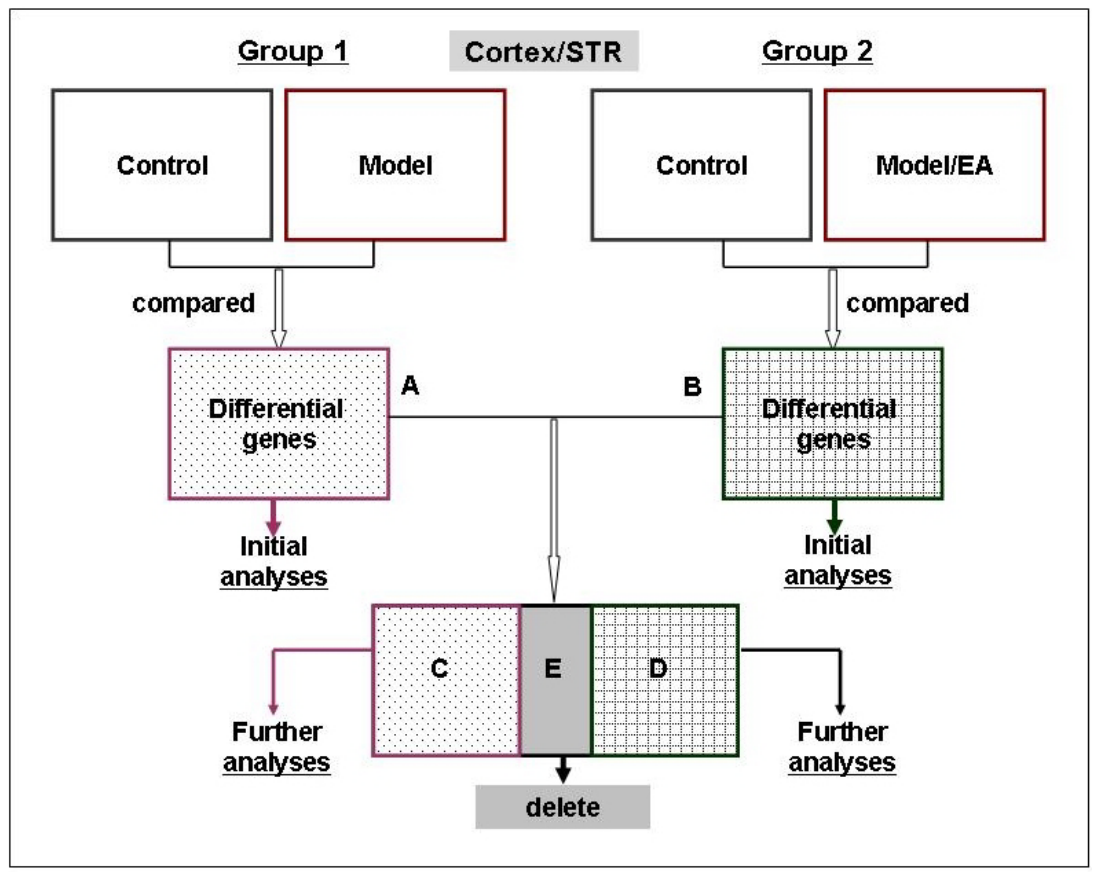

Figure 1. Flow chart comparing the two series. 
overlay the Cortex results of 4 and 5 weeks, 345 mutual genes were detected for further analysis (data not shown). Then, 944 differential transcripts (456 up-regulated and 488 down-regulated, $\mathrm{p}<0.05$ ) were used in the $4 \mathrm{~W}$, but not in the 5W, model group, and 567 differential transcripts (273 up-regulated and 294 down-regulated, $\mathrm{p}<0.05$ ) were used in the $5 \mathrm{~W}$, but not in the $4 \mathrm{~W}$, model group. The chips of STR showed 1039 differential transcripts (557 up-regulated and 482 down-regulated, $\mathrm{p}<0.05$ ) after 4 weeks of 6-OHDA treatment $(4 \mathrm{~W})$ and 913 differential transcripts (563 up-regulated and 350 down-regulated, $\mathrm{p}<0.05$ ) after 5 weeks $(5 \mathrm{~W})$ compared to control. To compare the 4-week and 5-week STR results, 405 mutual genes were detected for further analysis (data not shown). Then, 634 differential transcripts (301 up-regulated and 333 down-regulated, $\mathrm{p}<0.05$ ) were selected in the $4 \mathrm{~W}$, but not in the $5 \mathrm{~W}$, model group, and 508 differential transcripts (307 up-regulated and 201 down-regulated, $\mathrm{p}<0.05$ ) were selected in the $5 \mathrm{~W}$, but not in $4 \mathrm{~W}$, model group.

Discrete genes were a group of sporadic genes that could not compose a functional cluster. As the disease progresses, DG levels change. The differentially expressed transcripts in the cortex and STR were analyzed at two time points using DAVID 6.7. The percentage of DGs [DG/(DG + CG)] is shown in Figure 2. The analyzed data is shown in Table 1. The gene expression data are the result of the comparison of series A and B in Figure 1. According to a chi-square test, the constituent ratios were significantly different between the 4- and 5-week model groups in the cortex (each p value $<0.05$, Table 1). In the lesion STR groups, the constituent ratios of the $4 \mathrm{~W}$ and $5 \mathrm{~W}$ model groups were not significantly different from the control, but the ratios for the $4 \mathrm{~W}$ and $5 \mathrm{~W}$ models groups were significantly different with respect to up- and down-regulated genes (each p-value $<0.01$, Table 1).

Using DAVID Bioinformatics Resources 6.7, as described in Figure 1, we analyzed the aforementioned data according to the observed pattern of differentially expressed genes. We observed an interesting phenomenon while analyzing the data. It has been generally known that proteins do not work alone but instead assemble into complexes until their job is done [9]. To repair or response to the lesion in vivo, messenger RNA transcripts always respond earlier than the corresponding protein products. When we analyzed the genes that were differentially expressed in some encephalic regions using DAVID 6.7 (Functional Annotation Tool) [5] [6], we first removed genes without a name that corresponded to a chip ID. Then, the remaining genes where functionally annotated (see Table 1). Next, we defined discrete genes (DGs) as genes that could be a part of the functional

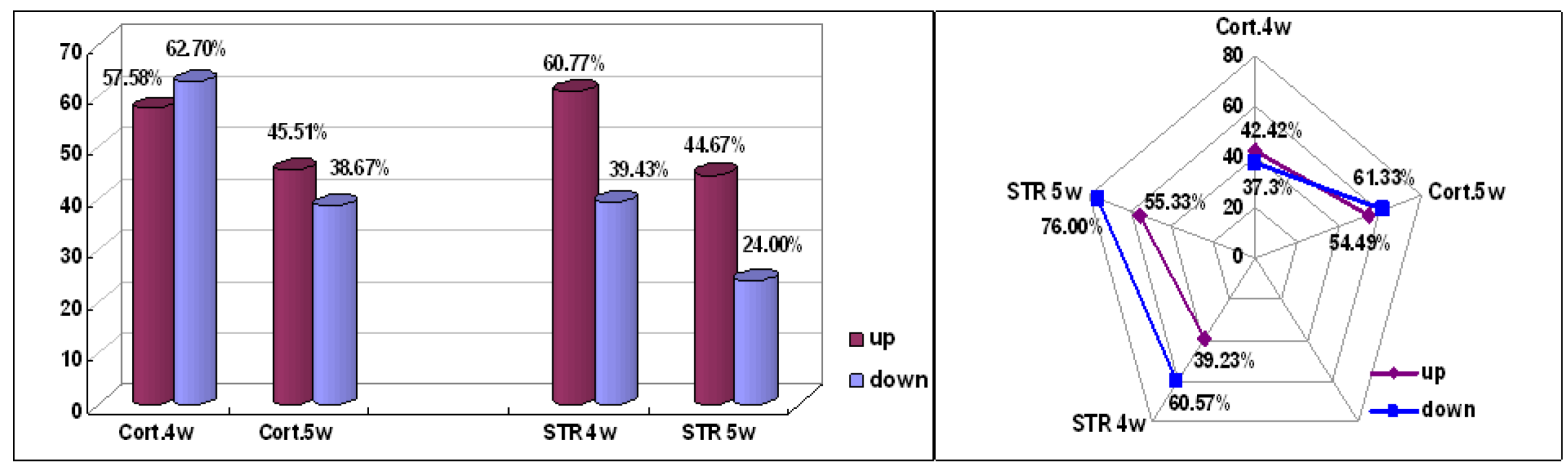

(a)

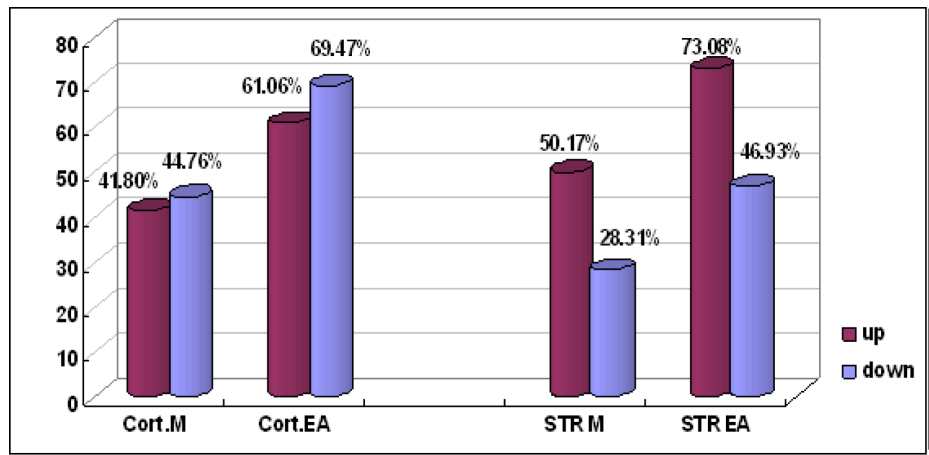

(c) (b)

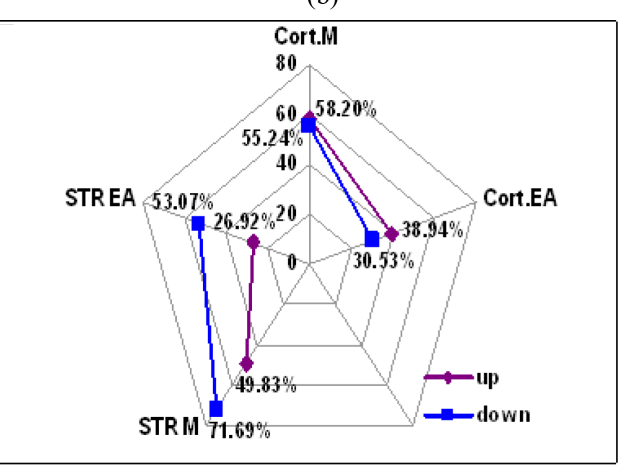

(d)

Figure 2. The discrete ratio and cluster ratio in the cortex and STR. 
Table 1. The percentages of DGs in functional annotation genes (Delete the unknown genes).

\begin{tabular}{|c|c|c|c|c|c|c|c|c|}
\hline \multirow{2}{*}{ Items } & M-Cort-4w & M-Cort-5w & M-Cort & EA-M-Cort & M-STR-4w & M-STR-5w & M-STR & EA-M-STR \\
\hline & (1) & (2) & (3) & (4) & (5) & (6) & (7) & (8) \\
\hline \multicolumn{9}{|c|}{ Respectively compared to control } \\
\hline Up-regulated genes & 414 & 273 & 273 & 662 & 395 & 383 & 383 & 238 \\
\hline DG in up-regulated genes & 173 & 147 & 147 & 265 & 187 & 188 & 188 & 61 \\
\hline The percentage of DGs & $41.79 \%$ & $53.85 \%$ & $53.85 \%$ & $40.03 \%$ & $47.34 \%$ & $49.09 \%$ & $49.09 \%$ & $25.63 \%$ \\
\hline Chi-square value & \multicolumn{2}{|c|}{9.6156} & \multicolumn{2}{|c|}{14.9637} & \multicolumn{2}{|c|}{0.2368} & \multicolumn{2}{|c|}{33.6225} \\
\hline p-value & \multicolumn{2}{|c|}{$P=0.001929$} & \multicolumn{2}{|c|}{$P=0.000110$} & \multicolumn{2}{|c|}{$P=0.626527$} & \multicolumn{2}{|c|}{$P=0.000000$} \\
\hline Down-regulated genes & 382 & 252 & 252 & 728 & 272 & 222 & 222 & 235 \\
\hline DG in down-regulated genes & 127 & 119 & 119 & 212 & 180 & 153 & 153 & 110 \\
\hline The percentage of DG & $33.25 \%$ & $47.22 \%$ & $47.22 \%$ & $29.12 \%$ & $66.18 \%$ & $68.92 \%$ & $68.92 \%$ & $46.81 \%$ \\
\hline Chi-square value & \multicolumn{2}{|c|}{12.489} & \multicolumn{2}{|c|}{27.4299} & \multicolumn{2}{|c|}{0.4179} & \multicolumn{2}{|c|}{22.8428} \\
\hline p-value & \multicolumn{2}{|c|}{$p=0.000409$} & \multicolumn{2}{|c|}{$p=0.000000$} & \multicolumn{2}{|c|}{$p=0.517987$} & \multicolumn{2}{|c|}{$p=0.000002$} \\
\hline \multicolumn{9}{|c|}{ Comparison between the two groups (for further analysis) } \\
\hline Up-regulated genes & 297 & 156 & 122 & 511 & 209 & 197 & 301 & 156 \\
\hline DG in up-regulated genes & 126 & 85 & 71 & 199 & 82 & 109 & 150 & 42 \\
\hline The percentage of DG & $42.42 \%$ & $54.49 \%$ & $58.20 \%$ & $38.94 \%$ & $39.23 \%$ & $55.33 \%$ & $49.83 \%$ & $26.92 \%$ \\
\hline Chi-square value & \multicolumn{2}{|c|}{5.9834} & \multicolumn{2}{|c|}{14.9222} & \multicolumn{2}{|c|}{10.5422} & \multicolumn{2}{|c|}{22.1373} \\
\hline p-value & \multicolumn{2}{|c|}{$p=0.014441$} & \multicolumn{2}{|c|}{$p=0.000112$} & \multicolumn{2}{|c|}{$p=0.001167$} & \multicolumn{2}{|c|}{$p=0.000003$} \\
\hline Down-regulated genes & 311 & 181 & 143 & 619 & 175 & 125 & 166 & 179 \\
\hline DG in down-regulated genes & 116 & 111 & 79 & 189 & 106 & 95 & 119 & 95 \\
\hline The percentage of DG & $37.30 \%$ & $61.33 \%$ & $55.24 \%$ & $30.53 \%$ & $60.57 \%$ & $76 \%$ & $71.69 \%$ & $53.07 \%$ \\
\hline Chi-square value & \multicolumn{2}{|c|}{24.0081} & \multicolumn{2}{|c|}{31.1211} & \multicolumn{2}{|c|}{7.845} & \multicolumn{2}{|c|}{12.6671} \\
\hline $\mathrm{p}$-value & \multicolumn{2}{|c|}{$p=0.000001$} & \multicolumn{2}{|c|}{$p=0.000000$} & $p=0$. & 05096 & $p=0$ & 00372 \\
\hline
\end{tabular}

Compared serials: (1) and (2); (3) and (4); (5) and (6); (7) and (8); M: Model group; Cort: cortex; STR: striatum; EA: electro-acupuncture; 4/5W: $4 / 5$ weeks.

analytical system (Functional Annotation Chart, DAVID 6.7) but were not found to have a statistically significant (i.e., with a p-value $\leq 1)$ functional annotation. These genes were neglected during analysis but in fact proved very important for explaining the level of disease progression. Their products varied predictably over time, and DG expression reliably predicted the stage of disease progression. That is, DGs showed little to no clustering for some functions. Correspondingly, the genes in the output results were defined as cluster genes (CGs).

Based on our observation of gene expression in different phases, we considered higher discrete ratios (the percentage DGs versus the sum of DGs and CGs) to be directly correlated to increasingly disordered corresponding patho-tissues. When we compared the differentially expressed genes at the two time points (4 weeks and 5 weeks), the up-regulated and the down-regulated transcripts compared to control were separated first, and then after analysis using DAVID 6.7, we found that the discrete ratio from the 5-week model increased not only in the cortex but also in the STR and included both up-regulated and down-regulated gene. When we deleted the mutual genes and compared the differentially expressed genes in the $4 \mathrm{~W}$ and $5 \mathrm{~W}$ model groups (see Figure 2(a) and Figure 2(b)), the same tendency toward an increase in the discrete ratio was observed in the 5W model groups for the cortex and for STR. For the lesioned STR, the discrete ratio was higher at 5 weeks than at 4 weeks when compared to results from the two model groups (see Table 1, p $<0.01$ ).

\subsection{Differentially Expressed Transcripts and DG Levels in the Cortex and STR between the PD Model Rats and the 100-Hz EA Group}

The Model group was analyzed 5 weeks after 6-OHDA injections into the unilateral MFB. The EA group was 
treated using 100-Hz EA for 4 weeks (EA treatment was applied during weeks 2 through 5 after the injection of 6-OHDA). The Cortex chips showed 912 differential transcripts (507 up-regulated and 405 down-regulated, $\mathrm{p}<$ 0.05 ) in the model group (Model) and 2369 differential transcripts (1280 up-regulated and 1089 down-regulated, $\mathrm{p}<0.05$ ) in the 100-Hz EA group (EA) compared to the control (a). To compare the Cortex results of the Model and EA groups, 483 mutual genes were detected for further analysis (data not shown). Then, 429 differential transcripts (212 up-regulated and 217 down-regulated, $\mathrm{p}<0.05$ ) were used in the Model group, but not in the EA group, and 1886 differential transcripts (985 up-regulated and 901 down-regulated, $p<0.05$ ) were used in the EA group, but not in the Model group (b). The STR chips showed 913 differential transcripts (563 up-regulated and 350 down-regulated, $\mathrm{p}<0.05$ ) in the Model group and 740 differential transcripts (387 up-regulated and 353 down-regulated, $\mathrm{p}<0.05$ ) in the EA group compared to the control (c). To compare the Cortex results of the Model and EA groups, 204 mutual genes were detected for further analyses (data not shown). Then, 709 differential transcripts (445 up-regulated and 264 down-regulated, $\mathrm{p}<0.05$ ) were selected in the Model group, but not in the EA group, and 536 differential transcripts (269 up-regulated and 267 down-regulated, $\mathrm{p}<0.05$ ) were selected in the EA group, but not in the Model group (d).

At the same time, we analyzed effective therapeutic measures for PD, such as electro-acupuncture (EA). EA, especially high-frequency EA, has been frequently used as an alternative therapy for PD and has been reportedly effective for alleviating motor symptoms in patients [10]-[13]. However, the physiological and biological mechanism behind its effectiveness is not completely understood. We used acupuncture points DAZHUI (GV14) and BAIHUI (GV21) for the treatment of model PD rats [13] [14]. Apomorphine-induced (0.5 mg/kg) motor disorder symptoms were significantly improved after EA treatment, but dopamine (DA) levels did not increase as significantly as in our previous results [13]. Results from similar approaches have suggested that the effect of acupuncture is comprehensive, systemic and poorly understood [13] [14]. Also, studies have indicated a robust association between brain gene expression in individuals (honey bees) and naturally occurring behavioral plasticity [15].

Both in the comparison between the model and EA-treated group and in the comparison between those groups and the control group, our results showed that the discrete ratio of the cortex and STR were significantly decreased after EA treatment (see Table 1, p < 0.005). Figure 2(d) show that the discrete ratio of down-regulated genes in the STR is higher than that in the corresponding cortex group. It suggested that increasingly down-regulated genes in the model STR were isolated and had difficult working effectively (belong to DGs), but that a significant decrease of the discrete ratio resulting from EA treatment helped to order and regulate the internal environment of the STR. Despite EA treatment, the STR was severely impaired; however, EA still is a good alternative therapy for PD because of the global regulation it provides. High-frequency EA can improve motor function by decreasing the discrete ratio of corresponding tissue and increasing the cluster ratio (see Figure 2(c) and Figure 2(d)).

\subsection{The Conclusions from the Percentage of DGs and CGs}

- In the impaired (damaged) samples, the higher the discrete ratio, the more serious was the damage in the region. In contrast, the higher cluster ratio, the more favorable was disease progression.

- The percentage of discrete or cluster genes can be used to determine the severity of PD.

- If possible, the discrete or cluster ratios calculated by analyzing differential gene expression can be used to assess therapeutic treatments.

- The value of the discrete or cluster ratios can be used to compare microarray data across similar clinical samples. In particular, when we analyzed clinical gene regulation data, we needed to know the state of disease progression among the PD patients in order to group them accordingly. The discrete ratio is more convenient for grouping patients. More importantly, the discrete ratio may be used to assess treatment efficacy: therapeutic treatments with lower ratios could be potential treatments for both multi-genic and sporadic PD.

Our results suggest that a new theoretical parameter of microarray analysis can be used to evaluate the extent disease progression in this complicated disorder. Additionally, the decrease in the discrete ratio after treatment with $100-\mathrm{Hz}$ EA may contribute to the behavioral improvement in PD model rats. However, development and deployment of EA for clinical evaluation and trials requires a detailed and complete strategy, which is beyond the scope of this paper.

\section{Figures and Tables}

Using David 6.7, we analyzed the normalized microarray data, described here. Group 1 and 2 were two compa- 
rable series consisting of either the Model groups at 4 and 5 weeks or Model and EA groups (Cortex or STR). First, we compared each group with their corresponding control (A and B). Second, the data between A and B were compared even further. The mutual genes were deleted $(\mathrm{E})$, and the differential genes between $\mathrm{A}$ and $\mathrm{B}$ are shown as $\mathrm{C}$ and $\mathrm{D}$.

The differentially expressed transcripts in the cortex and STR were analyzed at two time points (Delete the mutual genes, $\mathrm{A}$ and $\mathrm{B}$ ) or at Model and EA groups (Delete the mutual genes, $\mathrm{C}$ and $\mathrm{D}$ ). The entire analyzed results are shown in Results and Table 1. The percentage of CGs [CG/(DG + CG)] is shown in A and C, and the percentage of DGs [DG/(DG + CG)] is shown in B and D (each p-value $<0.01$, Table 1).

\section{Acknowledgements}

This work was supported by the National Basic Research Program of China (2011CB504100), the National Natural Science Foundation of China (31200811), and the "215" Talents Projects in Health System of Beijing (2013-3-096). There is no conflict of interest involved in this study.

\section{References}

[1] Zhu, Y., Shen, X. and Pan, W. (2009) Network-Based Support Vector Machine for Classification of Microarray Samples. BMC Bioinformatics, 10, S21. http://dx.doi.org/10.1186/1471-2105-10-S1-S21

[2] Henney, A. and Superti-Furga, G. (2008) A Network Solution. Nature, 455, 730-731. http://dx.doi.org/10.1038/455730a

[3] Yunger, S., Rosenfeld, L., Garini, Y. and Shav-Tal, Y. (2010) Single-Allele Analysis of Transcription Kinetics in Living Mammalian cells. Nature Methods, 7, 631-633. http://dx.doi.org/10.1038/nmeth.1482

[4] Braak, H., Del Tredici, K., Rüb, U., de Vos, R.A., Jansen Steur, E.N. and Braak, E. (2003) Staging of Brain Pathology Related to Sporadic Parkinson's Disease. Neurobiology of Aging, 24, 197-211. http://dx.doi.org/10.1016/S0197-4580(02)00065-9

[5] Dennis Jr., G., Sherman, B.T., Hosack, D.A., Yang, J., Gao, W., Lane, H.C., et al. (2003) DAVID: Database for Annotation, Visualization, and Integrated Discovery. Genome Biology, 4, 3. http://dx.doi.org/10.1186/gb-2003-4-5-p3

[6] Huang, D.W., Sherman, B.T. and Lempicki, R.A. (2009) Systematic and Integrative Analysis of Large Gene Lists Using DAVID Bioinformatics Resources. Nature Protocols, 4, 44-57. http://dx.doi.org/10.1038/nprot.2008.211

[7] Moran, L.B., Croisier, E., Duke, D.C., Kalaitzakis, M.E., Roncaroli, F., Deprez, M., et al. (2007) Analysis of AlphaSynuclein, Dopamine and Parkin Pathways in Neuropathologically Confirmed Parkinsonian Nigra. Acta Neuropathologica, 113, 253-263. http://dx.doi.org/10.1007/s00401-006-0181-6

[8] Huo, L.R., Liang, X.B., Li, B., Liang, J.T., He, Y., Jia, Y.J., et al. (2012) The Cortical and Striatal Gene Expression Profile of $100 \mathrm{hz}$ Electroacupuncture Treatment in 6-Hydroxydopamine-Induced Parkinson's Disease Model. Evid Based Complement Alternat Med, 2012, 908439. http://dx.doi.org/10.1155/2012/908439

[9] Abbott, A. (2002) The Society of Proteins. Nature, 417, 894-896. http://dx.doi.org/10.1038/417894a

[10] Cristian, A., Katz, M., Cutrone, E. and Walker, R.H. (2005) Evaluation of Acupuncture in the Treatment of Parkinson's Disease: A Double-Blind Pilot Study. Mov Disord, 20, 1185-1188. http://dx.doi.org/10.1002/mds.20503

[11] Shulman, L.M., Wen, X., Weiner, W.J., Bateman, D., Minagar, A., Duncan, R., et al. (2002) Acupuncture Therapy for the Symptoms of Parkinson's Disease. Mov Disord, 17, 799-802. http://dx.doi.org/10.1002/mds.10134

[12] Jeon, S., Kim, Y.J, Kim, S.T., Moon, W., Chae, Y., Kang, M., et al. (2008) Proteomic Analysis of the Neuroprotective Mechanisms of Acupuncture Treatment in a Parkinson's Disease Mouse Model. Proteomics, 8, 4822-4832. http://dx.doi.org/10.1002/pmic.200700955

[13] Jia, J., Sun, Z., Li, B., Pan, Y., Wang, H., Wang, X., et al. (2009) Electro-Acupuncture Stimulation Improves Motor Disorders in Parkinsonian Rats. Behav Brain Res, 205, 214-218. http://dx.doi.org/10.1016/j.bbr.2009.06.024

[14] Liu, X.Y., Zhou, H.F., Pan, Y.L., Liang, X.B., Niu, D.B., Xue, B., et al. (2004) Electro-Acupuncture Stimulation Protects Dopaminergic Neurons from Inflammation-Mediated Damage in Medial Forebrain Bundle-Transected Rats. Exp Neurol, 189, 189-196. http://dx.doi.org/10.1016/j.expneurol.2004.05.028

[15] Whitfield, C.W., Cziko, A.M. and Robinson, G.E. (2003) Gene Expression Profiles in the Brain Predict Behavior in Individual Honey Bees. Science, 302, 296-299. http://dx.doi.org/10.1126/science.1086807 